\title{
A COUNTEREXAMPLE TO THE INFINITY VERSION OF THE HYERS AND ULAM STABILITY THEOREM
}

\author{
EMANUELE CASINI AND PIER LUIGI PAPINI
}

(Communicated by Andrew M. Bruckner)

\begin{abstract}
Hyers and Ulam proved a stability result for convex functions, defined in a subset of $\mathfrak{R}^{n}$. Here we give an example showing that their result cannot be extended to those functions defined in infinite-dimensional normed spaces. Also, we give a positive result for a particular class of approximately convex functions, defined in a Banach space, whose norm satisfies the so-called convex approximation property.
\end{abstract}

\section{INTRODUCTION}

In this paper we discuss the following problem: let $\Delta$ be a convex subset of a Banach space $X$. Consider an arbitrary $\varepsilon$-convex function $f: \Delta \rightarrow \mathfrak{R}$, that is, a function that satisfies for every $x, y \in \Delta$ and every $\lambda \in[0,1]$ the inequality:

$$
f(\lambda x+(1-\lambda) y) \leq \lambda f(x)+(1-\lambda) f(y)+\varepsilon .
$$

Is it true that there exists a convex function $g: \Delta \rightarrow \mathfrak{R}$ such that $|g(x)-f(x)| \leq$ $K \varepsilon \forall x \in \Delta$, where $K$ is a constant depending only on $X$ ? A positive answer was given by Hyers and Ulam [HU] (see also [Gr, C]) in the case $X=\mathfrak{R}^{n}$ (with any norm!); the best known estimates are with $K_{n}=\min \left(M_{n}, L_{n}\right)$ where $M_{n}=\left(n^{2}+3 n\right) /(4 n+4)$ and $L_{n}=m / 2$ for $2^{m-1} \leq n<2^{m}$ (see also [Ge] for a discussion concerning these constants and related questions). We will show that in the infinite-dimensional case the stability theorem of [HU] does not hold. Also we shall give a positive answer when the space $X$ and the functions satisfy some additional properties. For midpoint convex functions a counterexample is known [Ge]; for a positive result concerning $\eta$-approximately convex functions, which are also $\varepsilon$-subadditive, see $[\mathrm{K}]$.

\section{Counterexample}

Let $X$ be a Banach space. We denote by $B(X)$ (resp. $\left.B_{r}(X)\right)$ the unit ball (resp. ball of radius $r$ ) of $X$, i.e., $B(X)=\{x \in X:\|x\| \leq 1\}$ (resp.

Received by the editors November 20, 1991.

1991 Mathematics Subject Classification. Primary 26B25; Secondary 46B99, 52A27.

Key words and phrases. Approximately convex functions, Hyers and Ulam theorem, convex approximation property.

During the preparation of the paper the authors were supported by grants from GNAFA-CNR and National Research Group "Functional Analysis" (M.U.R.S.T.). 
$\left.B_{r}(X)=\{x \in X:\|x\| \leq r\}\right)$, and if $A$ is a subset of $X$, by co $A$ its convex hull.

We say that the set $A \subseteq X$ satisfies the condition $\mathbf{C}^{\mathbf{0}}(\varepsilon)$ if $x, y \in A$ implies $(x+y) / 2 \in A+B_{\varepsilon}(X)$. We say that the set $A$ satisfies the condition $\mathbf{c}^{0}(\varepsilon)$ if $x, y \in A$ and $\lambda \in[0,1]$ implies $\lambda x+(1-\lambda) y \in A+B_{\varepsilon}(X)$.

Let $l_{1}$ be the Banach space of absolutely convergent sequences with the usual norm (that we will indicate by $\left.\|x\|_{1}\right)$. We will denote by $\left\{e_{i}\right\}$ the standard basis of $l_{1}$. Let $C^{+}=\left\{x \in l_{1}: x_{i} \geq 0\right\}$, the positive cone of $l_{1}$, and $B^{+}=C^{+} \cap B\left(l_{1}\right)$. For $p \in(0,1)$ set $S_{p}=\left\{x \in B^{+}: \sum_{i=1}^{+\infty} x_{i}^{p} \leq 1\right\}$. We need the following two propositions: the first one is in [L], but we give the proof since the paper is not very accessible, the second is in [CP].

Proposition 1. The set $S_{p}$ satisfies condition $\mathbf{C}^{\mathbf{0}}\left(2^{1 / p-1}-1\right)$.

Proof. Let $x, y \in S_{p}$ such that $\sum_{i=1}^{+\infty} x_{i}^{p}=\sum_{i=1}^{+\infty} y_{i}^{p}=1$. Then we have

$$
\begin{aligned}
\inf _{z \in S_{p}}\left\|\frac{x+y}{2}-z\right\|_{1} & =\inf _{\sum_{i=1}^{+\infty} z_{i}^{p}=1} \sum_{i=1}^{+\infty}\left|\frac{x_{i}+y_{i}}{2}-z_{i}\right| \\
& =\sum_{\sum_{i=1}^{+\infty} z_{i}^{p}=12 z_{i} \leq x_{i}+y_{i}} \sum_{i=1}^{+\infty}\left(\frac{x_{i}+y_{i}}{2}-z_{i}\right) .
\end{aligned}
$$

For positive numbers, $s^{p}+t^{p}=z^{p}$ implies $s+t \leq z$; thus

$$
\inf _{\sum_{i=1}^{+\infty} z_{i}^{p}=1} \sum_{i=1}^{+\infty}\left(\frac{x_{i}+y_{i}}{2}-z_{i}\right) \leq \inf _{\sum_{i=1}^{+\infty} s_{i}^{p}+t_{i}^{p}=1} \sum_{i=1}^{+\infty}\left(\frac{x_{i}}{2}-s_{i}\right)+\left(\frac{y_{i}}{2}-t_{i}\right) .
$$

Now let $x$ and $y$ be in $S_{p}$; define $x^{\prime}, y^{\prime}$ (still in $S_{p}$ and with disjoint supports) as follows:

$$
x_{2 n}^{\prime}=0, \quad x_{2 n-1}^{\prime}=x_{n}, \quad y_{2 n-1}^{\prime}=0, \quad y_{2 n}^{\prime}=y_{n} .
$$

Then, by using (2.1) and (2.2), we obtain

$$
\inf _{z \in S_{p}}\left\|\frac{x+y}{2}-z\right\|_{1} \leq \inf _{z \in S_{p}}\left\|\frac{x^{\prime}+y^{\prime}}{2}-z\right\|_{1},
$$

so we can suppose that $x$ and $y$ have disjoint supports. With this restriction, letting $z_{i}=\left(x_{i}+y_{i}\right) / 2^{1 / p}$ we obtain

$$
\begin{aligned}
\inf _{z \in S_{p}}\left\|\frac{x+y}{2}-z\right\|_{1} & \leq\left(\frac{1}{2}-\frac{1}{2^{1 / p}}\right) \sum_{i=1}^{+\infty}\left(x_{i}+y_{i}\right) \\
& \leq\left(\frac{1}{2}-\frac{1}{2^{1 / p}}\right)\left(\sum_{i=1}^{+\infty}\left(x_{i}+y_{i}\right)^{p}\right)^{1 / p}=2^{1 / p-1}-1 .
\end{aligned}
$$

Since $x$ and $y$ are arbitrary, $S_{p}$ satisfies condition $\mathbf{C}^{\mathbf{0}}\left(2^{1 / p-1}-1\right)$.

Proposition 2 (see [CP]). If $A$ satisfies $\mathbf{C}^{\mathbf{o}}(\varepsilon)$ then $A$ satisfies $\mathbf{c}^{\mathbf{0}}(2 \varepsilon)$.

So, in particular, $S_{p}$ satisfies condition $\mathbf{c}^{\mathbf{0}}\left(2^{1 / p}-2\right)$.

Now let us give the counterexample. For each $p \in(0,1)$, let $f_{p}: B^{+} \subset l^{1} \rightarrow$ $\Re$ be defined in the following way: $f_{p}(x)=\operatorname{dist}\left(x, S_{p}\right)=\inf _{z \in S_{p}}\|x-z\|_{1}$. First of all we will prove that $f_{p}$ is a $\left(2^{1 / p}-2\right)$-convex function. Take $\eta>0$, 
$x, y \in B^{+}$, and $z_{1}, z_{2} \in S_{p}$ such that $\left\|x-z_{1}\right\|_{1} \leq f_{p}(x)+\eta$ and $\left\|y-z_{2}\right\|_{1} \leq$ $f_{p}(y)+\eta$; then, by using Proposition 2, we obtain

$$
\begin{aligned}
f_{p}(\lambda x+(1-\lambda) y) & =\inf _{z \in S_{p}}\|\lambda x+(1-\lambda) y-z\|_{1} \\
\leq & \quad \inf _{z \in S_{p}}\left(\lambda\left\|x-z_{1}\right\|_{1}+(1-\lambda)\left\|y-z_{2}\right\|_{1}\right. \\
& \left.\quad+\left\|\lambda z_{1}+(1-\lambda) z_{2}-z\right\|_{1}\right) \\
\leq & \leq \lambda f_{p}(x)+(1-\lambda) f_{p}(y)+\eta+\inf _{z \in S_{p}}\left\|\lambda z_{1}+(1-\lambda) z_{2}-z\right\|_{1} \\
& \leq \lambda f_{p}(x)+(1-\lambda) f_{p}(y)+\eta+2^{1 / p}-2 .
\end{aligned}
$$

The conclusion follows from the arbitrariness of $\eta$.

Now suppose that there exists $K$ such that there exists a convex function $g: B^{+} \rightarrow \mathfrak{R}$ satisfying $\left|f_{p}(x)-g(x)\right| \leq K\left(2^{1 / p}-2\right)$. Take $\bar{p} \in(0,1)$ such that $K\left(2^{1 / \bar{p}}-2\right) \leq 1 / 4$. Let

$$
x_{n}=(\underbrace{1 / n, \ldots, 1 / n}_{n}, 0,0, \ldots)
$$

we have (since $\left.f_{p}\left(e_{i}\right)=0\right)$

$$
f_{\bar{p}}\left(x_{n}\right) \leq \frac{1}{4}+g\left(x_{n}\right) \leq \frac{1}{4}+\sum_{i=1}^{n} \frac{g\left(e_{i}\right)}{n} \leq \frac{1}{4}+\sum_{i=1}^{n} \frac{f_{\bar{p}}\left(e_{i}\right)+1 / 4}{n}=\frac{1}{2} .
$$

This is a contradiction, since an easy calculation shows that $f_{\bar{p}}\left(x_{n}\right)=1-$ $n^{1-1 / \bar{p}} \rightarrow 1$ as $n \rightarrow \infty$.

Remark 1. The finite-dimensional version of this counterexample shows that, at least asymptotically, the constants $K_{n}$ that appear in [C] are best possible. In fact, define $H_{n}$ as the best constant such that for every convex subset $\Delta$ of $\mathfrak{R}^{n}$ and for every $\varepsilon$-convex function $f$ on $\Delta$ there exists a convex function $g$ on $\Delta$ such that $|g(x)-f(x)| \leq H_{n} \varepsilon$ for every $x \in \Delta$. Consider $S_{p}^{n}=\left\{x \in \mathfrak{R}^{n}\right.$ : $\left.x_{i} \geq 0, \sum_{i=1}^{n} x_{i}^{p} \leq 1\right\}$, and define, as before, $f_{p}(x)=\inf _{z \in S_{p}^{n}}\|x-z\|_{1}$. Then, if $x_{n}=(1 / n, \ldots, 1 / n)$, proceeding in the same way as before, we obtain

$$
\begin{aligned}
1-n^{1-1 / p} & =f_{p}\left(x_{n}\right) \leq H_{n}\left(2^{1 / p}-2\right)+g\left(x_{n}\right) \\
& \leq H_{n}\left(2^{1 / p}-2\right)+\sum_{i=1}^{n} \frac{g\left(e_{i}\right)}{n} \\
& \leq H_{n}\left(2^{1 / p}-2\right)+\sum_{i=1}^{n} \frac{f_{p}\left(e_{i}\right)+H_{n}\left(2^{1 / p}-2\right)}{n} \\
& =2 H_{n}\left(2^{1 / p}-2\right) .
\end{aligned}
$$

This implies

$$
H_{n} \geq \frac{1-n^{1-1 / p}}{4\left(2^{1 / p-1}-1\right)} \sim \frac{\log _{2} n}{4} \quad \text { as } p \rightarrow 1^{-} .
$$

Remark 2. Now consider the Hilbert space $l_{2}=\left\{\left\{x_{n}\right\}: \sum_{n=1}^{+\infty} x_{n}^{2}<+\infty\right\}$ with the usual norm. Then the previous example works, also, if one thinks of $B^{+}$as a subset of $l_{2}$. Notice that the convex set $B^{+}$is a convex subset of $l_{2}$ (since algebraically $l_{1} \subset l_{2}$ ) and, also, it is a closed subset in $l_{2}$. Take, in fact, a 
sequence $\left\{x_{n}\right\} \subset B^{+}$such that $x_{n} \rightarrow x$ in the $l_{2}$ norm. Then $\left\{x_{n}\right\}$ converges to $x$ in the weak-topology of $l_{2}$ and so it converges coordinatewise. But, also, as a bounded sequence in $l_{1}$ it has a subnet converging in the weak*-topology of $l_{1}$ (and in particular coordinatewise) to an element of $l_{1}$. So $x$ belongs to $l_{1}$, thus to $B^{+}$.

Remark 3. As the previous remark pointed out, the stability problem is (in some sense) independent of the norm-topology of the Banach space in which the domain of our $\varepsilon$-convex functions lie. A way to relate the norm to the functions is to ask that they must satisfy some lipschitz condition. Notice that the functions $f_{p}$ considered in our counterexample are 1-lip, since it follows easily, from the definition of distance, that $\left|f_{p}(x)-f_{p}(y)\right| \leq\|x-y\|_{1}$. The same condition is not satisfied if we think of our example embedded in $l_{2}$. In fact, as a simple calculation shows, for any fixed $h$ our functions are not $h$-lip, for every $p$, in the $l_{2}$-norm. This will follow directly from Theorem 1 of the next section.

\section{A POSITIVE RESUlt}

In this section, we prove that the construction of our counterexample is possible since $l_{1}$ lacks a convexity property called the "convex approximation property" (C.A.P. for short). We will say that a Banach space $X$ has C.A.P. if for every $\varepsilon>0$ and $r>0$, there exists an integer $p=p(\varepsilon, r)$ such that for every $A \subseteq B_{r}(X)$ we have $\operatorname{co} A \subseteq \operatorname{co}_{p} A+B_{\varepsilon}(X)$, where $\operatorname{co}_{p} A=\{x \in X: x \in$ $\left.\sum_{i=0}^{p} \alpha_{i} x_{i}, x_{i} \in X, \alpha_{i} \geq 0, \sum_{i=0}^{p} \alpha_{i}=1\right\}$. In other words, each element of $\operatorname{co} A$ can be $\varepsilon$-approximated by a convex combination of no more than $p$ vectors of $A$. We will say that $X$ is $B$-convex [P] if there exist constants $c>0$, $p>1$ such that for every $n$ and all independent random variables $g_{1}, \ldots, g_{n}$ with values in $X$ we have

$$
E\left(\left\|\sum_{i=1}^{n} g_{i}\right\|_{X}^{p}\right) \leq c^{p} \sum_{i=1}^{n} E\left\|g_{i}\right\|_{X}^{p} .
$$

It is easy to construct a sequence of independent random variables with values in $l_{1}$ such that (3.1) does not hold. This implies that $l_{1}$ is not $B$-convex and so it does not have C.A.P. since we have the following result of Bruck [B].

Proposition 3. A Banach space $X$ has C.A.P. if and only if it is B-convex. Moreover, in this case, there exist constants $c>0, q>1$ depending on $X$ and $r$ such that, for every $A \subseteq B_{r}(X)$, we can choose $p$ (in the definition of C.A.P.) via $p \leq c \varepsilon^{q /(1-q)}$.

The main result is the following:

Theorem 1. Let $X$ a B-convex Banach space, $h>0$, and $\Delta$ a bounded convex subset of $X$. Then, for every $f: \Delta \rightarrow \mathfrak{R}$, which is $\varepsilon$-convex and $h$-lip, there exists (for $\varepsilon$ sufficiently small) a constant $K$ (depending on $X, h$, and $\operatorname{diam}(\Delta)$ ) such that there exists a convex function $g: \Delta \rightarrow \Re$ satisfying $|f(x)-g(x)| \leq K \varepsilon \lg _{2} \varepsilon$.

We will use the following lemma, the proof of which can be found in several papers and in [C] with the best known constants. 
Lemma 1. Let $X$ be a $B$-convex Banach space and $f: \Delta \subset X \rightarrow \mathfrak{R}$ an $\varepsilon$-convex function. Then for $x_{0}, \ldots, x_{p} \in \Delta, \alpha_{0}, \ldots, \alpha_{p} \in[0,1], \alpha_{1}+\cdots+\alpha_{p}=1$, and all integers $p$, we have $f\left(\sum_{i=0}^{p} \alpha_{i} x_{i}\right) \leq \sum_{i=0}^{p} \alpha_{i} f\left(x_{i}\right)+2 K_{p} \varepsilon$. (For the definition of $K_{n}$ see the introduction.)

Proof of Theorem 1. Suppose that $\Delta \subseteq B_{r}(X)$ and let $d=\operatorname{diam}(\Delta)$. We take $Z=X \oplus R$ with $\|z\|=\|(x, y)\|=\|x\|+|y|(x \in X, y \in R)$. It is easy to show that $Z$ is a $B$-convex Banach space since $X$ is $B$-convex, and so it has C.A.P. Since $f$ is $h$-lip then $f$ is bounded on $\Delta$. Let $m=\inf _{x \in \Delta} f(x)$, $M=\sup _{x \in \Delta} f(x)$, and $H_{0}=\{z \in Z: z=(x, y), x \in \Delta, f(x) \leq y \leq M\}$. We have to show that $H_{0}$ is a bounded subset of $Z$. In fact, if we take $z_{1}, z_{2} \in H_{0}$ we have

$$
\left\|z_{1}-z_{2}\right\|=\left\|x_{1}-x_{2}\right\|+\left|y_{1}-y_{2}\right| \leq d+M-m ;
$$

but as a consequence of the lipschitz condition on $f$ we have $M-m \leq h d$, so $\operatorname{diam}\left(H_{0}\right) \leq(1+h) d$.

Take $\eta>0$. Then there exists an integer $p$ such that if $z \in \operatorname{co} H_{0}$ there exist $z_{0}, \ldots, z_{p} \in H_{0}$ and $\alpha_{0}, \ldots, \alpha_{p}$ so that

$$
\left\|z-\sum_{i=0}^{p} \alpha_{i} z_{i}\right\|=\left\|x-\sum_{i=0}^{p} \alpha_{i} x_{i}\right\|+\left|y-\sum_{i=0}^{p} \alpha_{i} y_{i}\right| \leq \eta .
$$

Then by using Lemma 1

$$
\begin{aligned}
f(x) & =f(x)-f\left(\sum_{i=0}^{p} \alpha_{i} x_{i}\right)+f\left(\sum_{i=0}^{p} \alpha_{i} x_{i}\right) \\
& \leq\left|f(x)-f\left(\sum_{i=0}^{p} \alpha_{i} x_{i}\right)\right|+\sum_{i=0}^{p} \alpha_{i} f\left(x_{i}\right)+2 K_{p} \varepsilon \\
& \leq h \| x-\sum_{i=0}^{p} \alpha_{i} x_{i}||+\left|\sum_{i=0}^{p} \alpha_{i} y_{i}-y\right|+y+2 K_{p} \varepsilon \\
& \leq h^{\prime} \eta+y+2 K_{p} \varepsilon \quad\left(h^{\prime}=\max (h, 1)\right) .
\end{aligned}
$$

Taking into account the upper bound for $p$ given in Proposition 3 and the value of the constant $K_{n}$, we obtain (for $p$ sufficiently large, that is, for $\eta$ small)

$$
f(x) \leq h^{\prime} \eta+\left(1+\lg _{2} c \eta^{q /(1-q)}\right) \varepsilon+y .
$$

Since $\eta$ was arbitrary, we can choose: $\eta=q \varepsilon /(1-q) h^{\prime} \lg _{2} e$ (this is the minimum point of the function $\left.g(\eta)=h^{\prime} \eta+\left(1+\lg _{2} c \eta^{q /(1-q)}\right) \varepsilon\right)$. Then we obtain

$$
f(x) \leq 2 K \varepsilon \lg _{2} \varepsilon+y
$$

for some negative constant $K$. Now define, for $x \in \Delta, g_{0}(x)=\inf \{y \in R$ : $\left.(x, y) \in \operatorname{co} H_{0}\right\}$. Then by (3.2) we have $f(x)-2 K \varepsilon \lg _{2} \varepsilon \leq g_{0}(x) \leq f(x)$. It is not hard to prove that $g_{0}$ is a convex function, and if we put $g(x)=$ $g_{0}(x)+K \varepsilon \lg _{2} \varepsilon$ we obtain

$$
f(x)-K \varepsilon \lg _{2} \varepsilon \leq g(x) \leq f(x)+K \varepsilon \lg _{2},
$$

which concludes the proof. 


\section{REFERENCES}

[B] R. E. Bruck, On the convex approximation property and the asymptotic behavior of nonlinear contractions in Banach spaces, Israel J. Math. 38 (1981), 304-314.

[CP] E. Casini and P. L. Papini, Almost convex sets and best approximation, Ricerche Mat. (to appear).

[C] P. W. Cholewa, Remarks on the stability of functional equations, Aequationes Math. 27 (1984), 76-86.

[Ge] R. Ger, Almost approximately convex functions, Math. Slovaca 38 (1988), 61-78; Errata: 423.

[Gr] J. W. Green, Approximately convex functions, Duke Math. J. 19 (1952), 499-504.

[HU] D. H. Hyers and S. M. Ulam, Approximately convex functions, Proc. Amer. Math. Soc. 3 (1952), 499-504.

[K] Z. Kominek, On approximately subadditive functions, Demonstratio Math. 23 (1990), 155160.

[L] J.-O. Larsson, Studies on the geometrical theory of Banach spaces: part 5: Almost convex sets in Banach spaces of type $p, p>1$, Ph.D. Thesis, Uppsala Univ., 1987.

[P] G. Pisier, Sur les espaces de Banach qui ne contiennent pas uniformement de $l_{n}^{1}, \mathrm{C} . \mathrm{R}$. Acad. Sci. Paris Sér. A 277 (1973), 991-994.

Dipartimento di Matematica, Universitá di Bologna, Piazza Porta S. Donato, 5, I40127 BOLOGNA, ITALY

E-mail address, P. L. Papini: PAPINI@DM.UNIBO.IT 